University of Rhode Island

DigitalCommons@URI

Open Access Master's Theses

2017

\title{
Circadian Rhythm: A Functional Connection Between SHP and DEC1 Transcription Factor
}

Marek Matczynski Marczak

University of Rhode Island, mmmarczak@yahoo.com

Follow this and additional works at: https://digitalcommons.uri.edu/theses

\section{Recommended Citation}

Marczak, Marek Matczynski, "Circadian Rhythm: A Functional Connection Between SHP and DEC1 Transcription Factor" (2017). Open Access Master's Theses. Paper 1092.

https://digitalcommons.uri.edu/theses/1092

This Thesis is brought to you for free and open access by DigitalCommons@URI. It has been accepted for inclusion in Open Access Master's Theses by an authorized administrator of DigitalCommons@URI. For more information, please contact digitalcommons-group@uri.edu. 
CIRCADIAN RHYTHM: A FUNCTIONAL CONNECTION

BETWEEN SHP AND DEC1 TRANSCRIPTION FACTOR

MAREK MATCZYNSKI MARCZAK

A THESIS SUBMITTED IN PARTIAL FULFILLMENT OF THE

REQUIREMENTS FOR THE DEGREE OF

MASTER OF SCIENCE

IN

PHARMACEUTICAL SCIENCES

UNIVERSITY OF RHODE ISLAND

2017 
MASTER OF SCIENCE IN PHARMACEUTICAL SCIENCES THESIS

$\mathrm{OF}$

MAREK MARCZAK

\title{
APPROVED:
}

Thesis Committee:

Major Professor

\author{
Bingfang Yan \\ Ruitang Deng \\ Joel Chandlee
}

Nasser H. Zawia

DEAN OF THE GRADUATE SCHOOL

UNIVERSITY OF RHODE ISLAND

2017 


\begin{abstract}
Circadian rhythms ensure physiological processes to be coordinated with daily changes of the surrounding. Circadian rhythm misalignment has been increasingly recognized to pose health risk for a wide range of diseases, particularly metabolic disorders. The liver maintains metabolic homeostasis and express many circadian genes, such as the genes encoding differentiated embryo chondrocyte-1 (DEC1) and small heterodimer partner (SHP). DEC1 is established to repress transcription through class B E-box elements, and SHP belongs to the superfamily of nuclear receptors and has multiple Ebox elements in its promoter. Importantly, DEC1 and SHP are expressed in an inverse oscillating manner. The present study was performed to test the hypothesis that the SHP gene is a target gene of DEC1. In cotransfection experiments, we have demonstrated that DEC1 repressed the SHP promoter and attenuated the transactivation of the classic circadian activator complex of Clock/Bmall. Site-directed mutagenesis, electrophoretic mobility shift assay and chromatin immunoprecipitation established that the repression was achieved through the E-box in the proximal promoter. Overexpression of DEC1 led to decreased expression of SHP. In horse serum-shocked cells (induction of circadian rhythms), the widely used epileptic agent valproate inversely altered the expression of DEC1 and SHP. Both DEC1 and SHP are involved in energy balance and valproate is known to induce hepatic steatosis. Our findings collectively establish that DEC1 constitutes the negative loop of the SHP oscillating expression and that the DEC1-SHP pathway is intimately involved in energy homeostasis with profound pathophysiologic significance.
\end{abstract}




\section{ACKNOWLEDGMENTS}

I would first like to thank my thesis advisor and mentor for the previous 4 years, Dr. Bingfang Yan of the College of Pharmacy at the University of Rhode Island. I have gained tremendous experience under Dr. Yan's tutelage and appreciate the time and effort he has invested in helping me reach my goal. The guidance in maneuvering through my initial projects that did not yield meaningful results, but more importantly the support during the past 2 years in completing the project to our expectations, I truly could not have done this alone. I also would like to thank Dr. Yan's wife, Dongfang Yan, for the technical assistance on the weekends, helping troubleshoot any problems I may have incurred in my techniques performing experiments. Additionally, I would like to thank all the members of Yan Lab for their continued support and assistance. Thank You Dr. Yi-Tzai Chen, Dr. Karen Ho, Yuanjun Shen, and Zhen Xu.

I would also like to acknowledge Dr. Ruitang Deng and Dr. Joel Chandlee from the University of Rhode Island for being a part of committee and for the valuable comments and suggested changes to my work though out the project.

Finally, I must thank my mother, Hanna Matczynska, for the constant support and the endless love and comfort provided during my time at the University of Rhode Island. This would not at all be possible without her. From the long nights spent in the lab to numerous days on end where I would not come home, she was always there to help me and push me towards my goals. I have looked up to her and the amazing accomplishments she has made throughout her life and just hope to one day to be as successful. I hope to be a source of pride for you, Dziekuje Mamo, Kocham Ciebie! I 
would also like to thank my grandmother, Aleksandra Matczynska, for the unending moral support all the way from Poland. When struggling or trying to work through problems, I could always call her. I would also like to thank arguably the most influential person in my life, my grandfather, Marek Matczynski. I hope he is looking down full of pride and I hope I am caring on his name as he would have liked me to. I want to thank my siblings, Tomek and Aleksandra Marczak. I have pushed myself to do the best I can, trying to be a good role model and inspire them to be even greater. I cannot wait to see what you two are able to achieve in the future.

Thank you to all my friends for the constant support, and for providing me some relief when the stress of work would get to me. I love you all and thank you from the bottom of my heart.

Marek Matczynski Marczak 


\section{PREFACE}

The following manuscript has been formatted for publication in the Journal of Biological Chemistry. It will be submitted for publication in the coming months. 


\section{TABLE OF CONTENTS}

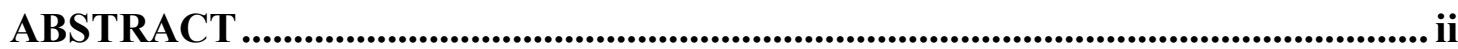

ACKNOWLEDGMENTS .............................................................................................. iii

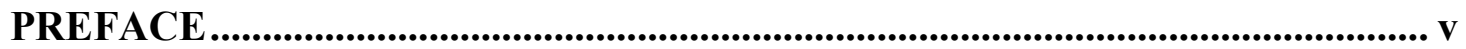

TABLE OF CONTENTS............................................................................. vi

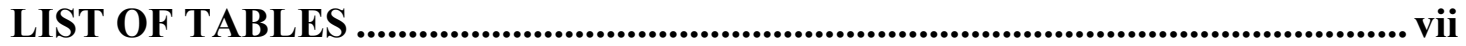

LIST OF FIGURES ........................................................................................... viii

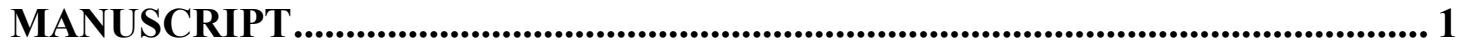




\section{LIST OF TABLES}

TABLE

PAGE

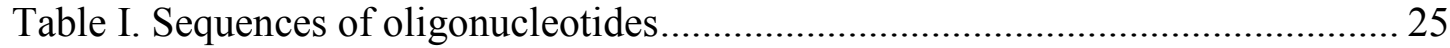




\section{LIST OF FIGURES}

FIGURE

PAGE

Figure 1. Regulation of SHP-luc and mPer1-luc by Clock/Bmal1, DEC1 or in

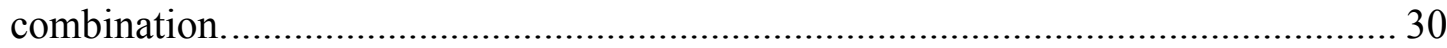

Figure 2. Identification and characterization of the E-box element in DEC1 binding .31

Figure 3. Inverse expression between DEC1 and SHP 32 


\title{
MANUSCRIPT \\ CIRCADIAN RHYTHMICITY: A FUNCTIONAL \\ CONNECTION BETWEEN DIFFERENTIATED EMBRYONIC CHONDROCYTE-1 \\ (DEC1)
}

\section{AND SMALL HETERODIMER PARTNER (SHP) ${ }^{1}$}

\author{
Marek M Marczak and Bingfang Yan² \\ Department of Biomedical and Pharmaceutical Sciences \\ University of Rhode Island \\ Kingston, RI 02881
}

\section{Address}

Correspondence to:

Dr. Bingfang Yan

Department of Biomedical and Pharmaceutical

Sciences

Center for Integrated Drug Development

University of Rhode Island

Kingston, RI 02881

Tel. (401) 874-5032

Fax. (401) 874-5048

E-mail: byan@uri.edu

Running Title: $\quad$ Repression of SHP by DEC1

Key words: $\quad$ DEC1, BHLHE40

SHP, NROB2

Circadian rhythm 


\section{FOOTNOTES}

1 This work was supported by National Institutes of Health Grants R01GM61988 and R01EB018748.

2 To whom correspondence should be addressed.

${ }^{3}$ Abbreviation used: Bmal1, Brain and muscle ARNT-like 1; Clock, circadian locomotor output cycles kaput; Cry, Cryptochrome; CYP7A1, Cytochrome P450 enzyme cholesterol 7a-hydroxylase; DEC, Differentiated embryo chondrocyte; DMEM, Dulbecco's modified Eagle's medium; EMSA, Electrophoretic mobility shift assay; FXR, Farnesoid X receptor; Per, Period; PCR, Polymerase chain reaction; RT-PCR, Reverse transcription-polymerase chain reaction; SCN, suprachiasmatic nucleus; SHP, small heterodimer partner. 


\section{ABSTRACT}

Circadian rhythms ensure physiological processes to be coordinated with daily changes of the surrounding. Circadian rhythm misalignment has been increasingly recognized to pose health risk for a wide range of diseases, particularly metabolic disorders. The liver maintains metabolic homeostasis and express many circadian genes, such as the genes encoding differentiated embryo chondrocyte-1 (DEC1) and small heterodimer partner (SHP). DEC1 is established to repress transcription through class B E-box elements, and SHP belongs to the superfamily of nuclear receptors and has multiple E-box elements in its promoter. Importantly, DEC1 and SHP are expressed in an inverse oscillating manner. The present study was performed to test the hypothesis that the SHP gene is a target gene of DEC1. In cotransfection experiments, we have demonstrated that DEC1 repressed the SHP promoter and attenuated the transactivation of the classic circadian activator complex of Clock/Bmal1. Site-directed mutagenesis, electrophoretic mobility shift assay and chromatin immunoprecipitation established that the repression was achieved through the E-box in the proximal promoter. Overexpression of DEC1 led to decreased expression of SHP. In horse serum-shocked cells (induction of circadian rhythms), the widely used epileptic agent valproate inversely altered the expression of DEC1 and SHP. Both DEC1 and SHP are involved in energy balance and valproate is known to induce hepatic steatosis. Our findings collectively establish that DEC1 constitutes the negative loop of the SHP oscillating expression and that the DEC1-SHP pathway is intimately involved in energy homeostasis with profound pathophysiologic significance. 


\section{INTRODUCTION}

Circadian rhythms are physiological changes that follow a 24-h cycle [1]. These changes ensure physiological processes to be coordinated with daily fluctuations of the environment [1-3]. Circadian rhythm misalignment has been increasingly recognized to pose health risk for a wide range of diseases including obesity, diabetes, cardiovascular diseases and stroke [4-7]. Mammals have central and peripheral circadian clocks [8]. The central clock is located in the suprachiasmatic nucleus (SCN) of the hypothalamus and is entrained by such environmental cues as the light-dark cycle $[9,10]$. Through the retinohypothalamic tract, the SCN receives photic input signals and generates rhythms, which subsequently synchronize multiple peripheral clocks through neural and humoral signaling [9-12]. While each organ has its own clock, the liver clock is the most studied peripheral clocks, particularly related to food cues [13-16].

Circadian rhythms are controlled by a group of core clock genes such as Clock (circadian locomotor output cycles kaput) and Bmal1 (brain and muscle ARNT-like 1) $[8,17]$. Clock and Bmal1 form a heterodimer and transactivate through E-Box elements the Per (Period-1 and 2) and Cry genes (Cryptochrome-1 and 2). Per and Cry proteins in turn interact with Clock/Bmal1 and attenuate their own transactivation $[5,8,17]$. In addition to Clock/Bmal1, we and other investigators have demonstrated that DEC transcription factors, differentiated embryo chondrocyte-1 and 2, are strong E-box binding proteins [18-20]. However, binding to E-box by DEC transcription factors leads to efficacious repression of the target genes including Per1. DEC1 and DEC2 
are both central and peripheral oscillators [15]. In particular, DEC1 has been recognized to play fundamental roles in orchestrating metabolism and resetting the liver clock. Indeed, DEC1 is one of the most sensitive genes in responding to food cues. A 30 min feeding significantly induces the hepatic expression of DEC1 [15].

In addition to DEC1, there are several other well-characterized hepatic circadian genes such as the gene encoding small heterodimer partner gene (SHP) $[5,21,22]$. SHP has been established as a major regulator of diverse metabolic pathways, particularly in bile acid synthesis, lipid metabolism, glucose homeostasis and liver fibrogenesis [23]. Structurally, SHP belongs to the superfamily of nuclear receptors [23, 24]. However, it lacks the DNA binding domain, thus is referred to as an atypical nuclear receptor. SHP has been shown to interact with nuclear receptors and/or compete for co-factors (e.g., co-activators or co-repressor), delivering potent regulatory activities at the transcriptional level [23]. On the other hand, many nuclear receptors have been shown to support the induction of SHP in response to endobiotics such as bile acids. In addition to nuclear receptors, the circadian complex of Clock/Bmal1 is a potent transactivator of the SHP gene [23]. The transactivation is achieved through the element CACGTG, a special type of E-box recognized by DEC transcription factors [23]. In mice, the expression of DEC1 and SHP is inversely oscillating [25, 26].

The present study was performed to test the hypothesis that the SHP gene is a target of DEC1. Cotransfection experiments showed that DEC1 efficaciously repressed the SHP promoter reporter and attenuated the transactivation of SHP by the Clock/Bmal1 complex. A set of molecular experiments including chromatin immunoprecipitation established that the repression was achieved through the E-box in the proximal promoter. Overexpression of DEC1 led to decreased expression of SHP, and valproate 
inversely altered the oscillating expression of DEC1 and SHP. These findings collectively establish that DEC1 constitutes the negative loop of the SHP oscillating expression and that the DEC1-SHP pathway is involved in energy homeostasis with profound pathophysiologic significance.

\section{MATERIALS AND METHODS}

\section{$\underline{\text { Plasmid }}$}

Expression constructs of Bmal1 and Clock were gifts of Dr. Marina P Antoch of the Cleveland Clinic [27]. The Per1 promoter reporter (Per1-luc) was a gift of Dr. Joseph S. Takahashi of Northwestern University [28]. The SHP-2.2 Luc reporter was a gift of Dr. Hueng-Sik Choi of Keimyung University [29]. The 5' deletion mutants of the SHP reporter were prepared by inserting a Nhe I-Hind III fragment into the pGL3 basic vector. These fragments were generated by PCR with primers as described in Table

I. The SHP mutant reporter with a disrupted E-box (E1) was prepared with the same approach but the mutations were introduced in the forward primer (Table I). The DEC1 expression construct and its mutants (deletion or substitution) were described elsewhere $[18,30]$. All constructs were subjected to sequencing analysis.

\section{$\underline{\text { Reporter activity and cotransfection }}$}

Cotransfection was performed, essentially as described previously [31, 32]. Cells (293T) were plated in 24-well plates in DMEM media supplemented with $10 \%$ fetal bovine serum at a density of $1.6 \times 10^{5}$ cells per well. Transfection was conducted by lipofection with Lipofectamine and Plus Reagents (Thermo Fisher Scientific, Waltham, 
MA). Generally, the transfection mixtures contained DEC1 (0-100 ng) or a corresponding mutant construct (100 ng), a reporter plasmid (50 ng) and the pRL-null Renilla plasmid (5 ng) unless otherwise specified. Vector plasmid was used to equalize the amount of plasmid DNA for each transfection. The transfected cells were cultured for additional $24 \mathrm{~h}$, washed with PBS and resuspended in passive lysis buffer (Promega, Madison, WI). The lysed cells were subjected to 2 cycles of freezing/thawing. The reporter enzyme activities were assayed with a Dual-Luciferase Reporter Assay System (Promega). This system contained two substrates, which were used to determine the activity of two luciferases sequentially. The firefly luciferase activity, which represented the reporter gene activity, was initiated by mixing an aliquot of lysates $(20 \mu \mathrm{l})$ with Luciferase Assay Reagent II. Then the firefly luminescence was quenched and the Renilla luminescence was simultaneously activated by adding Stop \& Glo Reagent to the sample wells. The firefly luminescence signal was normalized based on the Renilla luminescence signal.

\section{Electrophoretic mobility shift assay (EMSA)}

DEC1 stably transfected cells with a tetracycline-inducible construct [33] were cultured in the presence or absence of tetracycline $(0.1 \mu \mathrm{g} / \mathrm{mL})$ for 24 . Cells were harvested and nuclear extracts were prepared with a nuclear extraction kit (Active Motif, Carlsbad, CA). The EMSA experiment was performed with Lightshift Chemiluminescent EMSA Kit (Thermo Fisher Scientific, Waltham, MA) as described previously [34]. The sense strand for SHP-187/162 (E1) was synthesized as labeled or non-labeled form (for competition). Nuclear protein $(5 \mu \mathrm{g})$ was mixed with binding buffer and then incubated with a double-stranded biotinylated probe $(0.1 \mathrm{pmol})$ on ice for $20 \mathrm{~min}$. In competition assays, nuclear extracts were first incubated with an unlabeled probe at a 50x excess 
for $30 \mathrm{~min}$ before addition of the labeled probe. For antibody-disruption assay, the nuclear extracts were first incubated with anti-DEC1 antibody on ice for $20 \mathrm{~min}$ and then with the labeled probe. As a positive control, the EMSA experiment was performed with a DEC2 E-box containing probe [18]. The protein-DNA complexes were resolved by non-denaturing polyacrylamide gel electrophoresis $(6 \%)$ and transferred onto a Biodyne ${ }^{\circledR}$ nylon membrane. The biotinylated probe was detected with Chemiluminescent Nucleic Acid Detection Module Kit (Thermo Fisher Scientific, Waltham, MA). The chemiluminescent signal was captured by myECL Imager (Thermo Fisher Scientific, Waltham, MA).

\section{Chromatin immunoprecipitation (ChIP)}

ChIP experiment was performed with Active Motif ChIP-IT Express kit, essentially described previously [31, 34]. HepG2 Cells were transfected with DEC1 (Flagtagged) by TransfeX (ATCC, Manassas, VA) for $24 \mathrm{~h}$, washed and underwent cross-linking for $15 \mathrm{~min}$ by $\mathbf{1 . 0 \%}$ formaldehyde at room temperature, and the cross-linking was terminated with glycine (final concentration of $125 \mathrm{mM}$ ). The soluble chromatins were prepared as described previously [31, 34]. Alternatively, DEC1 stably transfected cells were cultured in the presence of tetracycline $(0.1 \mu \mathrm{g} / \mathrm{ml})$ for $24 \mathrm{~h}$ and then processed as described for HepG2 cells. For ChIP experiment, chromatins were pre-cleared for $2 \mathrm{~h}$ at $4^{\circ} \mathrm{C}$ with protein $\mathrm{G}$ beads pre-treated with herring sperm DNA $(0.2 \mathrm{mg} / \mathrm{ml})$ and BSA $(0.5 \mathrm{mg} / \mathrm{ml})$. A fraction of the pre-cleared chromatins was stored at $-80^{\circ} \mathrm{C}$ for later use as an input. The pre-cleared chromatins were incubated with anti-Flag antibody (HepG2) or anti-DEC1 (stably transfected cells) for overnight at $4^{\circ} \mathrm{C}$. As a negative control, incubation was performed with pre-immune IgG. The antibodybound chromatins and DNA input as well as IgG control chromatins were 
analyzed by PCR for the presence of the genomic fragments containing the Ebox of interest, other E-boxes (not repressed by DEC1) with primers shown in Table I. The PCR was performed with Platinum Taq DNA polymerase for a total of 32 cycles at $94^{\circ} \mathrm{C}$ for $30 \mathrm{~s}, 58^{\circ} \mathrm{C}$ for $30 \mathrm{~s}$ and $68^{\circ} \mathrm{C}$ for $60 \mathrm{~s}$. A 3-min initial denaturation was performed.

\section{Suppressed expression of SHP by DEC1}

HepG2 cells were seeded in 8 -well chamber slides $\left(2 \times 10^{4}\right.$ cells/ well and cultured for $24 \mathrm{~h}$. Cells were then transfected with Flag-DEC1 (500 ng) or the vector by TransfeX reagent. The transfected cells were cultured for another $24 \mathrm{~h}$ and subsequently washed with ice cold PBS and then fixed with 4\% Paraformaldehyde for $10 \mathrm{~min}$ at $\mathrm{pH}$ 7.4. Cells were washed 3 times with PBS and permeabilization solution $(0.1 \%$ Triton X-100) was added for $10 \mathrm{~min}$. Chamber slides were incubated with $1 \% \mathrm{BSA}(2 \mathrm{mg} / \mathrm{mL})$ for $1 \mathrm{~h}$ to block nonspecific binding. The slides were incubated overnight with anti-SHP antibody ( $\mathrm{H}-160$, Santa Cruz Biotechnology, Dallas, TX) or anti-Flag antibody (M2, Sigma-Aldrich, St. Louis, MO) at a dilution of 1:200. The anti-SHP antibody was located by Alexa Fluor® 488 conjugated goat anti-rabbit lgG (green), whereas the anti-Flag antibody with Alexa Fluor ${ }^{\circledR} 555$ conjugated goat anti-mouse lgG (red). Both secondary

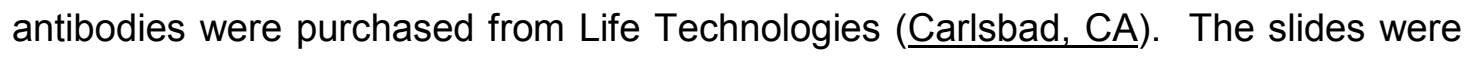
then mounted with ProLong Gold Antifade Mountant (Thermo Fisher). The mounting media contained 4',6-diamidino-2-phenylindole (DAPI) for staining nuclei (Blue). Cells were then imaged using confocal microscope. To provide more quantitative information, same experiments were performed in 6-well plates and the expression of SHP and DEC1 was determined by Western blotting. 
$\underline{\text { Regulated expression of DEC1 and SHP by valproate in serum-shocked circadian }}$ induction

HepG2 cells were seeded in 6- or 24-well plates at a density of $6 \times 10^{5}$ or $1.5 \times 10^{5}$ cells/well in low glucose DMEM with $10 \%$ delipided FBS. When the confluency reached $75 \%$, cells were shocked with media containing $50 \%$ horse serum for $2 \mathrm{~h}$. Thereafter, the shocked cells were cultured in normal media or the same media containing valproate at $2 \mathrm{mM}$. Cells were harvested at $6 \mathrm{~h}$ interval. The expression of DEC1 and SHP was determined by RT-qPCR with Taqman probes. The TaqMan assay identification numbers were: DEC1: Hs00186419_m1; SHP: Hs00222677_m1; GAPDH, 4352934E; and RNA polymerase II, Hs00172187_m1.

\section{Other analyses}

The anti-DEC1 antibody against a peptide derived from the $C$-terminus was described elsewhere [33]. Protein concentration was determined with BCA assay (Pierce) with bovine serum albumin as the standard. Data are presented as mean \pm SD of at least four separate experiments, except where results of blots are shown in which case a representative experiment is depicted in the figures. Statistical significance between 2 means was made according to 1-way ANOVA followed by a DUNCAN's multiple comparison test $(P<0.05)$.

\section{RESULTS}

Repression of the SHP promoter by DEC1

DEC1 and SHP are established to play critical roles in a wide range of biological activities including metabolic homeostasis $[23,35,36]$. Both DEC1 and SHP are 
circadian genes and their expression is inversely rhythmed [25, 26]. We have shown that DEC1 is a sequence-specific transcription factor that acts on Sp1 site as well as a specific type of E-box: CACAGT $[30,37]$. The SHP promoter and its immediate upstream sequence contain multiple E-boxes including a CACATG. We therefore hypothesized that DEC1 transcriptionally regulates the expression of SHP. To test this hypothesis, we first examined whether DEC1 represses the SHP promoter. Specifically, cotransfection experiments were performed with an SHP promoter luciferase reporter in in 293T cells. For comparison, a Per1 reporter was included. We and other investigators have demonstrated that Per 1 is a circadian gene and negatively regulated by DEC1 [18, 30].

As shown in Fig. 1, DEC1 repressed both SHP and Per1 reporters and the repression occurred in a dose-dependent manner. The repression was robust by as much as $90 \%$. Nevertheless, the SHP reporter was repressed to a greater extent than the Per1 reporter by $10-15 \%$ depending on the amount of DEC1 construct used for the transfection (Fig. 1A). We next tested whether DEC1 attenuates Clock/Bmal1transactivation of the SHP and Per1 promoters, as the Clock/Bmal1 heterodimer has been shown to regulate both DEC1 and SHP in a circadian manner $[23,30]$. Once again, cotransfection was performed. As shown in Fig. 1B, Clock/Bmal1 strongly transactivated both the SHP and Per1 reporters with the Per1 reporter being transactivated to a greater extent (7 versus 12 fold) (Fig. 1B). However, the transactivation of the SHP reporter was attenuated to a much greater extent than that of the Per1 reporter by DEC1. For example, DEC1 at $10 \mathrm{ng}$ attenuated the Clock/Bmal 1 transactivation of the SHP reporter by $97 \%$. In contrast, the Clock/Bmal1 transactivation of the Per1 reporter was attenuated by $55 \%$ only with the same amount 
of DEC1. We next tested whether the repression of the SHP promoter requires DNA binding. As shown in Fig. 1C, no repression was detected with all constructs except DEC1 (wild-type) and DEC1 1 56A. We have previously shown that substitution of the residue proline-56 with an alanine remained the ability for DEC 1 to bind to E-box and deliver repressive activity $[18,30]$. In contrast, substitution of the residue arginine-58 with a proline no longer bound to E-box element. These results conclude that DEC1 is a transcriptional repressor of SHP.

\section{Repression of the SHP promoter by DEC1 through the E-box in the proximal}

\section{promoter}

The proximal promoter of SHP is an E-box rich region and has as many as $7 \mathrm{E}$-box elements [29]. However, these elements differ slightly with 2 of them being CACCTG, and 1 of the following: CACTTG, CATCTG, CAGCTG, CAGGTG and CACGTG. To specify whether one or more of these elements support DEC1 repression, deletion and site-directed mutants of the SHP reporter were prepared and tested for the responsiveness to DEC1. Once again, DEC1 stably transfected cells were cultured in the presence or absence of tetracycline and then transfected with a reporter. As shown in Fig. 2A, all deletion SHP reporters were repressed except SHP-116Luc, suggesting that the E-box (E1: CACGTG) in the SHP-191Luc reporter supported the repression. To specify the role of this E-box in DEC1 repression, reporter SHP-191Luc was subjected to site-directed mutagenesis to selectively disrupt the E-box (CACGTG to AACGGG). As shown in Fig. 2A, disruption of this E-box completely attenuated DEC1mediated repression of the SHP promoter. 
We next tested whether this E-box interacted directly with DEC1. The DEC1-stable line was cultured in the presence or absence of tetracycline, and nuclear extracts were prepared. Double-stranded oligonucleotides harboring this E-box were synthesized and biotinylated. The labeled probe was incubated with the nuclear extracts and analyzed by EMSA. As shown in Fig. 2B, incubation with the extracts from the cells cultured in the presence of tetracycline yielded a shifted band. This band was not detected when incubation was performed with the extracts from the cell cultured without tetracycline (lane 11). The shifted band was competed completely by $50 \times$ unbiotinylated oligonucleotide (lane 5). A mutant of this oligonucleotide or oligonucleotides corresponding to other E-box elements showed no competitive activity (Fig. 2B, lanes 6-10). In addition, the shifted band disappeared by anti-DEC1 antibody (disrupted binding). As expected, a shifted band was detected with biotinylated oligonucleotide harboring an E-box derived from the DEC2 promoter [18]. In contrast to the disrupted binding with the SHP E-box (E1), the anti-DEC1 antibody caused the formation of a supershifted band (Right of Fig. 2B).

To determine whether DEC1 occupies the SHP promoter region that harbors this Ebox (i.e., E1), ChIP experiment was performed. To gain specificity, primers were designed to amplify three fragments: the binding $E$-box $(B E)$ fragment ( $E 1$ E-box); the other E-box (OE) fragment (other E-boxes but not binding) and the non E-box (NE) fragment (no E-box). As shown in Fig. 2C, chipped DNA showed the abundant presence of the BE-box fragment (labeled as lane 1) but not the other fragments. As expected, input DNA produced amplification of all three fragments (Right of Fig. 2C). It should be noted that pre-immune IgG was used as a control but did not enrich any fragments. The ChIP experiment was performed with HepG2 cells transfected with 
DEC1 (Flag-tagged) and DEC1 stably transfected cells. Same observation was made with both cells. 


\section{Effect of DEC1 on the expression of SHP}

The reporter assay, EMSA and ChIP experiments established that DEC1 is a transcriptional repressor of SHP. Next we tested whether DEC1 suppressed the expression of endogenous SHP. To test this possibility, HepG2 cells were transfected with DEC1 (Flag-tagged) or the corresponding vector, and the expression of SHP was determined. Both Western blotting and immunocytochemistry were used to determine the changes of SHP expression. As expected, transfection of DEC1 increased immunodetection of DEC1. Importantly, transfection of DEC1 decreased the expression of SHP (Right of Fig. 3A). The suppressed expression of SHP by DEC1 was confirmed by immunocytochemistry (Fig. 3A). Overall, transfection of DEC1 led to abundant Texas-Red staining (DEC1) accompanied by decreased FITC staining (SHP). Based on the overlay with DAPI staining (blue), both DEC1 and SHP are primarily present in the nuclei. It should be noted that the transfection was performed with TransfeX reagent, which delivered high transfection efficiency.

To complement the transfection study, we next tested whether SHP and DEC1 are inversely regulated for their oscillating expression by valproate, a widely used antiepileptic that was established to down-regulate SHP [38]. Importantly, valproate is a steatotic agent and both DEC1 and SHP are metabolic regulators. To mimic circadian rhythm, HepG2 cells were shocked by horse-serum and then treated with valproate. Cells were collected starting at $6 \mathrm{~h}$ after serum-shock and then at a $6 \mathrm{~h}$ interval. Total RNA was isolated and analyzed for the expression of SHP and DEC1. Both genes were expressed in a circadian manner and the patterns of the expression were inversed between these two genes (Fig. 3B). 


\section{DISCUSSION}

Normal circadian rhythms ensure physiological processes to be coordinated with daily changes of the environment [1-3]. Circadian rhythm misalignment has been increasingly recognized to pose health risk for a wide range of diseases [4-7]. The DEC1 and SHP genes are members of the liver clock and have been shown to play critical roles in metabolic homeostasis $[5,23,35,36]$. In this study, we have shown that the SHP gene is a target of DEC1. DEC1 efficaciously repressed the SHP promoter reporter and attenuated the transactivation of SHP by the Clock/Bmal 1 complex. Site-directed mutagenesis, EMSA and ChIP identified the E-box element in the SHP proximal promoter that supported the repression. Overexpression of DEC1 led to decreased expression of SHP. In horse serum-shocked cells (induction of circadian rhythms), the steatotic drug valproate inversely altered the oscillating expression of DEC1 and SHP.

These findings establish that DEC1 constitutes the negative loop of the SHP oscillating expression. It has been reported that the SHP gene is a target of the heterodimer Clock/Bmal1, a circadian activator complex. The Clock/Bmal1 and Per/Cry pairs are 
well-established pairs that generate clock outputs by the transcriptional/translational feedback loop [5]. The Clock/Bmal1 dimer transactivates the Per and Cry genes through E-box elements, and the Per/Cry interacts with Clock/Bmal1 and attenuates the Clock/Bmal1 transactivation of Per/Cry. In this study, we have shown that DEC1 repressed the SHP promoter through the same E-box transactivated by the Clock/Bmal1 dimer, suggesting that DEC1 and Clock/Bmal1 form the transcriptional feedback loop for the oscillating expression of SHP (Figs. 1A and B). Consistent with the notion, the expression of DEC1 and SHP is inversely oscillating $[25,26]$.

It remains to be determined whether the Per/Cry proteins negatively regulate the expression of SHP. Nonetheless, it is likely that DEC1 exerts a dominant repression, particularly on the oscillating expression of SHP. In this study, we have shown that DEC1 repressed both the SHP promoter and the Per1 promoter with the SHP promoter being repressed to a greater extent (Fig. 1A). Importantly, DEC1 at $10 \mathrm{ng}$ attenuated the Clock/Bmal1 transactivation of the SHP promoter by as much as $97 \%$ but only $55 \%$ on the transactivation of the Per1 promoter (Fig. 1B). In both cases, DEC1 exerted repressive activity by binding to E-box CACGTG. The SHP E-box is flanked by GTGC (5') and GGGT (3'), respectively, whereas the Per1 by TAGC and ACAG, respectively. It remains to be determined whether the differences in flanking sequences contribute to the differences in response to DEC1 repression. An early study demonstrated that the flanking sequences were important for interacting affinity with stra13, the mouse counterpart of DEC1 [26]. In this study, we have also shown that the antibody against DEC1 disrupted the shifted band with the SHP E-box (Fig. 2B). In contrast, we have shown that the same antibody caused a supershift of the DEC2 E-box and the Per1 Ebox $[19,30]$. 
The DEC1-SHP pathway likely plays critical roles in the synthesis of bile acids, particularly the circadian production of these endobiotics. While there are several pathways that have been shown to regulate the synthesis of bile acids, the pathway mediated by SHP and FXR (farnesoid X receptor) has been extensively studied for the bile acid-activated regulatory cascade $[5,23]$. This cascade is commonly referred as the bile acid negative feedback inhibition on the expression of the cytochrome P450 enzyme cholesterol 7a-hydroxylase (CYP7A1). CYP7A1 is the first and rate-limited enzyme in bile acid synthesis. Increased production of bile acids activates FXR, leading to the induction of SHP. Induction of SHP inactivates LRH-1 (liver receptor homolog-1) and HNF4a (hepatocyte nuclear factor $4 \alpha$ ). In this study, we have shown that DEC1 downregulated SHP, thus counteracting the feedback inhibition. Interestingly, the transcription factor DEC2 (functionally related to DEC1) reportedly repressed the expression of rat CYP7A1 through an E-box: CACATG [39]. This E-box is conserved in human and mouse based on a BLAST search. It remains whether DEC1 binds to this E-box and causes repression. Nevertheless, we have reported that DEC1 negatively regulated the expression of DEC2. It is likely that DEC1 de-represses CYP7A1 by downregulating SHP and DEC2.

The DEC1-SHP pathway likely serves as an important mechanism for lipid metabolism. Although there are exceptions, SHP is generally considered to be lipogenic whereas DEC1 is anti-lipogenic. SHP reportedly augmented the transactivation by PPAR $\gamma$ (peroxisome proliferator-activated receptor- $\gamma$ ), leading to marked lipid accumulation in the liver [7]. Likewise, transgenic expression of SHP induced liver steatosis [41]. Consistent with these observations, SHP null mice were protected against diet-induced 
obesity. DEC1, on the other hand, has been shown to repress lipogenic genes such as fatty acid synthase and inhibit adipogenesis $[42,43]$. Overexpression of DEC1 by viral transduction alleviated fatty liver phenotypes accompanied by suppressed expression of the lipogenic gene Srebp-1c (sterol regulatory element-binding protein 1c) [35]. Interestingly, SHP null mice supported higher induction of Srebp-1c in response to cholic acid treatment, suggesting that SHP is a repressor of Srebp-1c [44]. It is not clear whether the observed repression has a broad implication.

The DEC1-SHP pathway may have profound significance in carbohydrate homeostasis. Patients with type 2 diabetes had higher frequency of loss-of-function SHP mutants than those without type 2 diabetes (61.5 versus 28.1\%) [45]. In addition, SHP mutation carriers had significantly higher fasting plasma insulin levels than noncarriers [45]. In mice, knockout of SHP developed hepatic insulin resistance [46], and the antidiabetic metformin ameliorated cytokine-induced hepatic insulin resistance by inducing SHP [47]. These observations suggest that SHP positively regulates glucose homeostasis. In contrast, high glucose and high insulin significantly induced DEC1 [48, 49]. The induction of DEC1 was inhibited by LY294002, a strong inhibitor of phosphoinositide 3-kinases. Importantly, DEC1 protein and the activity of AMPK (5' AMP-activated protein kinase) showed an inverse circadian rhythm, and knockdown of DEC1 expression increased AMPK activity [50]. AMPK is known to regulate glucose homeostasis and prevent insulin resistance [51].

In summary, SHP belongs to the superfamily of nuclear receptors and has been established to exert a wide range of biological activities, particularly related to metabolic homeostasis. Many nuclear receptors and other transcription factors reportedly 
support the induction of SHP. On the other hand, the SHP gene is transactivated by the circadian complex of Clock/Bmal1. However, the negative loop of SHP oscillating expression remains unknown. In this study, we report that DEC1 repressed the SHP promoter and the repression was achieved through the E-box element in the proximal promoter region. We have also demonstrated that transfection of DEC1 led to decreased expression of SHP. In horse serum-shocked cells, the epileptic agent valproate (a steatotic drug) inversely altered the expression of DEC1 and SHP. Our findings collectively establish that DEC1 constitutes the negative loop of the SHP oscillating expression. Emerging evidence suggests that alterations on circadian systems are important risk factors for disease initiation and progression, and the expression of DEC1 and SHP is rapidly altered by many endobiotics and xenobiotics. Therefore, de-regulated expression of DEC1 and SHP genes likely alters normal circadian rhythms and contributes significantly to the pathogenesis of many diseases, particularly metabolic disorders.

\section{REFERENCE}

1. Millius A, Ueda HR (2017) Systems Biology-Derived Discoveries of Intrinsic Clocks. Front Neurol. 8:25. 
2. Riede SJ, van der Vinne V, Hut RA (2017) The flexible clock: predictive and reactive homeostasis, energy balance and the circadian regulation of sleep-wake timing. J Exp Biol. 220:738-749.

3. Dominoni DM, Borniger JC, Nelson RJ (2016) Light at night, clocks and health: from humans to wild organisms. Biol Lett. 12:20160015.

4. Touitou Y, Reinberg A, Touitou D (2017) Association between light at night, melatonin secretion, sleep deprivation, and the internal clock: Health impacts and mechanisms of circadian disruption. Life Sci. 173:94-106.

5. Ferrell JM, Chiang JY (2015) Circadian rhythms in liver metabolism and disease. Acta Pharm Sin B. 5:113-22.

6. Potter GD, Skene DJ, Arendt J, Cade JE, Grant PJ, Hardie LJ (2016) Circadian Rhythm and Sleep Disruption: Causes, Metabolic Consequences, and Countermeasures. Endocr Rev. 2016 37:584-608.

7. Kunze KN, Hanlon EC, Prachand VN, Brady MJ (2016) Peripheral circadian misalignment: contributor to systemic insulin resistance and potential intervention to improve bariatric surgical outcomes. Am J Physiol Regul Integr Comp Physiol. 311:R558-63.

8. Korenčič A, Košir R, Bordyugov G, Lehmann R, Rozman D, Herzel H (2014) Timing of circadian genes in mammalian tissues. Sci Rep. 4:5782.

9. Nakamura TJ, Takasu NN, Nakamura W (2016) The suprachiasmatic nucleus: agerelated decline in biological rhythms. J Physiol Sci. 66:367-74.

10. Kalsbeek A, Palm IF, La Fleur SE, Scheer FA, Perreau-Lenz S, Ruiter M, Kreier F, Cailotto C, Buijs RM (2006) SCN outputs and the hypothalamic balance of life. J Biol Rhythms. 21:458-69.

11. Besharse JC, McMahon DG (2016) The Retina and Other Light-sensitive Ocular Clocks. J Biol Rhythms. 31:223-43.

12. Tan E, Scott EM (2014) Circadian rhythms, insulin action, and glucose homeostasis. Curr Opin Clin Nutr Metab Care. 17:343-8.

13. Hirao A, Nagahama H, Tsuboi T, Hirao M, Tahara Y, Shibata S (2010) Combination of starvation interval and food volume determines the phase of liver circadian rhythm in Per2::Luc knock-in mice under two meals per day feeding. Am J Physiol Gastrointest Liver Physiol. 299:G1045-53.

14. Wu T, Yao C, Huang L, Mao Y, Zhang W, Jiang J, Fu Z (2015) Nutrients and Circadian Rhythms in Mammals. J Nutr Sci Vitaminol (Tokyo). 61 Suppl:S89-91.

15. Wu T, Ni Y, Kato H, Fu Z (2010) Feeding-induced rapid resetting of the hepatic circadian clock is associated with acute induction of Per2 and Dec1 transcription in rats. Chronobiol Int. 27:1-18. 
16. Oike H, Nagai K, Fukushima T, Ishida N, Kobori M (2011) Feeding cues and injected nutrients induce acute expression of multiple clock genes in the mouse liver. PLoS One. 6:e23709.

17. Challet E, Caldelas I, Graff C, Pevet P (2003) Synchronization of molecular clock by light- and food-related cues in mammals. Biol Chem 384:711-9.

18. Honma S, Kawamoto T, Takagi Y, Fujimoto K, Sato F, Noshiro M, Kato Y, Honma K. (2002) DEC1 and DEC2 are regulators of the mammalian molecular clock. Nature 419:841-4.

19. Li Y, Zhang H, Xie M, Ge S, Hu M, Yang D, Wan Y, Yan B (2003) DEC1 negatively regulates the expression of DEC2 through the E-box in the proximal promoter. J. Biol. Chem. 278:16899-907

20. St-Pierre B, Flock G, Zacksenhaus E, Egan SE (2002) Stra13 homodimers repress transcription through class B E-box elements. J Biol Chem. 277:46544-51.

21. Wang L, Liangpunsakul S (2016) Circadian clock control of hepatic lipid metabolism: role of small heterodimer partner (Shp). J Investig Med. 64:1158-61.

22. Wu N, Kim KH, Zhou Y, Lee JM, Kettner NM, Mamrosh JL, Choi S, Fu L, Moore DD (2016) Small Heterodimer Partner (NR0B2) Coordinates Nutrient Signaling and the Circadian Clock in Mice. Mol Endocrinol. 30:988-95.

23. Zhang $\mathrm{Y}$, Hagedorn $\mathrm{CH}$, Wang $\mathrm{L}$ (2011) Role of nuclear receptor SHP in metabolism and cancer. Biochim Biophys Acta. 1812:893-908.

24. Chiang JY. (2009) Bile acids: regulation of synthesis. J Lipid Res. 50:1955-66.

25. Oiwa A, Kakizawa T, Miyamoto T, Yamashita K, Jiang W, Takeda T, Suzuki S, Hashizume K (2007) Synergistic regulation of the mouse orphan nuclear receptor SHP gene promoter by CLOCK-BMAL1 and LRH-1. Biochem Biophys Res Commun. 353:895-901.

26. Grechez-Cassiau A, Panda S, Lacoche S, Teboul M, Azmi S, Laudet V, Hogenesch JB, Taneja R, Delaunay $F(2004)$ The transcriptional repressor STRA13 regulates a subset of peripheral circadian outputs. J Biol Chem. 279:1141-50.

27. Kondratov RV, Chernov MV, Kondratova AA, Gorbacheva VY, Gudkov AV, Antoch MP (2003) BMAL1-dependent circadian oscillation of nuclear CLOCK: posttranslational events induced by dimerization of transcriptional activators of the mammalian clock system. Genes Dev. 17:1921-32.

28. Wilsbacher LD, Yamazaki S, Herzog ED, Song EJ, Radcliff LA, Abe M, Block G, Spitznagel E, Menaker M, Takahash JS (2002) Photic and circadian expression of luciferase in mPeriod1-luc transgenic mice in vivo. Proc Natl Acad Sci USA. 99:48999.

29. Kim HJ, Kim JY, Kim JY, Park SK, Seo JH, Kim JB, Lee IK, Kim KS, Choi HS (2004) Differential regulation of human and mouse orphan nuclear receptor small heterodimer 
partner promoter by sterol regulatory element binding protein-1. J Biol Chem. 279:28122-31.

30. Li Y, Song X, Ma Y, Liu J, Yang D, Yan B (2004) DNA binding, but not interaction with $\mathrm{Bmal}$, is responsible for DEC1-mediated transcription regulation of the circadian gene Per1. Biochem J. 382:895-904.

31. Xiao D, Yang D, Guo L, Lu W, Charpentier M, Yan B (2013) Regulation of carboxylesterase-2 expression by p53 family proteins and enhanced anticancer activities among 5-fluorouracil, irinotecan and doxazolidine prodrug. Brit J Pharmacol. 168:1989-99.

32. Vachirayonsti $T$, Yan B (2016) MicroRNA-30c-1 suppresses the pregnane $X$ receptor by targeting the 3'-untranslated region and alters the expression of its target gene cytochrome P450 3A4. BBA-Gene Regulatory Mechanisms. 1859:1238-44.

33. Li Y, Zhang H, Xie M, Hu M, Ge S, Yang D, Wan Y, Yan B (2002) DEC1/STRA13/SHARP2 is abundantly expressed in colon carcinoma, antagonizes serum deprivation induced apoptosis and selectively inhibits the activation of procaspases. Biochem J. 367:413-22.

34. Vachirayonsti T, Yang D, Yan B (2015) Suppression of the pregnane X receptor during endoplasmic reticulum stress is achieved by down-regulating hepatocyte nuclear factor- $4 \alpha$ and up-regulating liver-enriched inhibitory protein. Toxicol Sci. 144:382-92.

35. Shen L, Cui A, Xue Y, Cui Y, Dong X, Gao Y, Yang H, Fang F, Chang Y (2014) Hepatic differentiated embryo-chondrocyte-expressed gene 1 (Dec1) inhibits sterol regulatory element-binding protein-1c (Srebp-1c) expression and alleviates fatty liver phenotype. J Biol Chem. 289:23332-42.

36. Shen M, Yoshida E, Yan W, Kawamoto T, Suardita K, Koyano Y, Fujimoto K, Noshiro M, Kato Y. (2002) Basic helix-loop-helix protein DEC1 promotes chondrocyte differentiation at the early and terminal stages. J Biol Chem. 277:50112-20.

37. Li Y, Xie M, Yang J, Yang D, Deng R, Wan Y, Yan B (2006) The expression of antiapoptotic protein survivin is transcriptionally upregulated by DEC1 primarily through multiple Sp1 binding sites in the proximal promoter. Oncogene. 25:3296-306.

38. Benet M, Guzmán C, Pisonero-Vaquero S, García-Mediavilla MV, SánchezCampos S, Martínez-Chantar ML, Donato MT, Castell JV, Jover R (2015) Repression of the nuclear receptor small heterodimer partner by steatotic drugs and in advanced nonalcoholic fatty liver disease. Mol Pharmacol. 87:582-94.

39. Noshiro M, Kawamoto T, Furukawa M, Fujimoto K, Yoshida Y, Sasabe E, Tsutsumi S, Hamada T, Honma S, Honma K, Kato Y (2004) Rhythmic expression of DEC1 and DEC2 in peripheral tissues: DEC2 is a potent suppressor for hepatic cytochrome P450s opposing DBP. Genes Cells. 9:317-29.

40. Nishizawa $H$, Yamagata $K$, Shimomura I, Takahashi $M$, Kuriyama $H$, Kishida $K$, Hotta K, Nagaretani H, Maeda N, Matsuda M, Kihara S, Nakamura T, Nishigori H, 
Tomura H, Moore DD, Takeda J, Funahashi T, Matsuzawa Y (2002) Small heterodimer partner, an orphan nuclear receptor, augments peroxisome proliferator-activated receptor gamma transactivation. J Biol Chem. 277:1586-92.

41. Boulias K, Katrakili N, Bamberg K, Underhill P, Greenfield A, Talianidis I (2005) Regulation of hepatic metabolic pathways by the orphan nuclear receptor SHP. EMBO J. 24(14):2624-33.

42. Ozaki N, Noshiro M, Kawamoto T, Nakashima A, Honda K, Fukuzaki-Dohi U, Honma S, Fujimoto K, Tanimoto K, Tanne K, Kato Y (2012) Regulation of basic helixloop-helix transcription factors Dec1 and Dec2 by RORa and their roles in adipogenesis. Genes Cells. 17:109-21.

43. lizuka K, Horikawa $Y$ (2008) Regulation of lipogenesis via BHLHB2/DEC1 and ChREBP feedback looping. Biochem Biophys Res Commun. 374:95-100.

44. Wang L, Lee YK, Bundman D, Han Y, Thevananther S, Kim CS, Chua SS, Wei P, Heyman RA, Karin M, Moore DD (2002) Redundant pathways for negative feedback regulation of bile acid production. Dev Cell. 2:721-31.

45. Yang Z, Wu S, Zheng T, Lu H, Ma X, Jia W, Hu R (2010) Gender-dependent penetrance of small heterodimer partner (SHP) gene deficiency in overweight/obese Chinese pedigrees. J Int Med Res. 38:142-9.

46. Park YJ, Kim SC, Kim J, Anakk S, Lee JM, Tseng HT, Yechoor V, Park J, Choi JS, Jang HC, Lee KU, Novak CM, Moore DD, Lee YK (2011) Dissociation of diabetes and obesity in mice lacking orphan nuclear receptor small heterodimer partner. J Lipid Res. 52:2234-44.

47. Kim YD, Kim YH, Cho YM, Kim DK, Ahn SW, Lee JM, Chanda D, Shong M, Lee $\mathrm{CH}$, Choi HS (2012) Metformin ameliorates IL-6-induced hepatic insulin resistance via induction of orphan nuclear receptor small heterodimer partner (SHP) in mouse models. Diabetologia. 55:1482-94.

48. Chen R, Wang Y, Ning R, Hu J, Liu W, Xiong J, Wu L, Liu J, Hu G, Yang J (2015) Decreased carboxylesterases expression and hydrolytic activity in type 2 diabetic mice through Akt/mTOR/HIF-1 $\alpha /$ Stra13 pathway. Xenobiotica. 45:782-93.

49. Yamada K, Kawata H, Shou Z, Mizutani T, Noguchi T, Miyamoto K (2003) Insulin induces the expression of the SHARP-2/Stra13/DEC1 gene via a phosphoinositide 3kinase pathway. J Biol Chem. 278:30719-24.

50. Sato F, Muragaki Y, Zhang Y (2015) DEC1 negatively regulates AMPK activity via LKB1. Biochem Biophys Res Commun. 467:711-6.

51. Nandipati KC, Subramanian S, Agrawal DK (2017) Protein kinases: mechanisms and downstream targets in inflammation-mediated obesity and insulin resistance. Mol Cell Biochem. 426:27-45. 
Table I. Sequences of oligonucleotides

\begin{tabular}{ll}
\hline Oligonucleotide & \multicolumn{1}{c}{ Sequence } \\
\hline Native promoter reporters [numbered according to Kim et al., J. Biol. Chem. (2004), \\
$279: 28122-31$ ] \\
SHP-572 Nhel \\
SHP-240 Nhel \\
SHP-190 Nhel & 5'-GTGAGCGGCAGGTGGCCCT-3' \\
SHP-117 Nhel & 5'-GTGATATCAGTGCCACGT-3' \\
SHP-190m Nhel & 5'-TGTCTGTGTGTTTTTTCA-3' \\
GTGATATCAGTGCAACGGGGGGTTCCCAATGCC-3' \\
SHP+30 HindIII & 5'- \\
& 5'-GGTTAGGGATCTGCTCTC-3' \\
EMSA & \\
SHP-187/162 (E1) & \\
SHP-242/217 (E2) & \\
SHP-280/255 (E4/5) & 5'-ATATCAGTGCCACGTGGGGTTCCCAA-3' \\
SHP-332/307 (E6) & 5'-GAGTGAGCGGCAGGTGGCCCTGTGCC-3' \\
SHP-347/322 (E7) & 5'- GCTGATTGTGCACCTGGGGCCTTGGT-3' \\
SHP-187/162m (E1) & 5'- CCAATGGGGACACCTGCTGATTGTGC-3' \\
DEC2 E-box & 5'- ATATCAGTGCAACGGGGGGTTCCCAA-3' \\
& 5'-TACGTTCCGCACGTGAGCTGGGTG-3' \\
ChIP & \\
E1 element sense & 5'-GGCCCTGTGCCCTGCACCGGC-3' \\
E1 element antisense & 5'-CTCATGGTTAGGGATCTGCTC-3' \\
Other E-box element sense & 5'-CCTAGTCTTTTGGCACACAA-3', \\
Other E-box element antisense & 5'-TCACCTCAGTCAATGAAGTGG-3' \\
Non-element sense & 5'-CAGAAATTCTTGTCACTGTTT-3' \\
Non-element antisense & 5'-CACACCTCTTTCATTTGATTA-3' \\
\hline
\end{tabular}


Fig. 1. Regulation of SHP-luc and mPer1-luc by Clock/Bmal1, DEC1 or in combination (A) Repression of SHP-LUC and Per1-LuC by DEC1 Cells (293T) were cultured in 24 -well plates at $\sim 80 \%$ confluence and transfected with a reporter construct (50 ng), the pRL-null Renilla (5 ng) and DEC1 (0-100 ng). Vector plasmid was used to equalize the amount of plasmid DNA for each transfection. The transfected cells were cultured for $24 \mathrm{~h}$, collected with PBS and resuspended in passive lysis buffer. The reporter enzyme activities were assayed with a Dual-Luciferase Reporter Assay System. The firefly luminescence signal was normalized based on the Renilla luminescence signal. The signal in the absence of DEC1 was recoded as $100 \%$. Statistical significance $(p<0.05)$ is denoted by a different letter of $a, b$ and $c$ or $x, y$ and z. (B) Attenuated Clock/Bmal1 activation by DEC1 Cells were transfected with a reporter construct (50 ng), the pRL-null Renilla (5 ng), Clock/Bmal1 (100 ng each labeled as C or B) and DEC1 (0-100 ng labeled as D) and the pRL-null Renilla (5 ng). Vector plasmid (V) was used to equalize the amount of plasmid DNA for each transfection. The reporter enzyme activities were assayed with a Dual-Luciferase Reporter Assay System and normalized based on the Renilla luminescence signal. The signal was expressed relatively to that in the absence of DEC1. Statistical significance $(p<0.05)$ is denoted by a different letter of $a, b$ and $c$ or $x, y$ and $z$. (C) Essentiality of DNA binding domain for DEC1 to repress SHP-LUC Cells (293T) were cultured in 24-well plates and transfected with DEC1 or a DEC1 mutant (100 ng), SHPLuc (50 ng) and the pRL-null Renilla (5 ng). Vector plasmid was used to equalize the amount of plasmid DNA for each transfection. After a $24 \mathrm{~h}$-incubation, cells were collected and analyzed for luciferase activities. Similarly, firefly luminescence signal was normalized based on the Renilla luminescence signal. 
Fig. 2. Identification and characterization of the E-box element in DEC1 binding (A) Identification of the E-box element in DEC1 binding DEC1 stably transfected cells were cultured in the presence or absence of tetracycline to modulate the expression of DEC1 (tetracycline inducible) for $24 \mathrm{~h}$. The cells were then transfected with a reporter construct (50 ng), the pRL-null Renilla (5 ng) and DEC1 (0-100 ng). The reporter constructs contained the 5' sequence of the SHP promoter at different length or a sequence with a disrupted E-box element. The transfected cells were cultured, collected and analyzed for luciferase activity as described above. Once again, the firefly luminescence signal was normalized based on the Renilla luminescence signal. The signal in the absence of DEC1 was recoded as $100 \%$ for each reporter. (B) EMSA analysis DEC1-stably transfected cells were cultured in the presence of absence of tetracycline $(0.1 \mu \mathrm{g} / \mathrm{ml})$ for $24 \mathrm{~h}$, and the nuclear extracts were isolated. For EMSA, nuclear extracts $(5 \mu \mathrm{g})$ were incubated with a biotinylated 187/162 (E1) probe for 20 min. In the competition assay, nuclear extracts were pre-incubated with the unlabeled element (50x) or oligonucleotides containing another E-box (not the same as the probe) for $30 \mathrm{~min}$, and then incubated with the biotinylated probe. In the disruption assay, nuclear extracts were incubated first with an antibody against DEC1 on ice for 20 min and then with the biotinylated probe. Nuclear extracts (labeled as " $n$ ") from cells cultured without tetracycline were used as a control (right lane of the left panel). For comparison, a probe derived from DEC2, known to interact with DEC1, was included. The protein-DNA complexes were electrophoretically resolved, transferred to a Biodyne ${ }^{\circledR}$ nylon membrane and located with streptavidin-conjugated horseradish peroxidase and chemiluminescent substrate. (C) ChIP analysis HepG2 cells were transfected with DEC1 (Flag-tagged) for $24 \mathrm{~h}$, washed and underwent cross-linking for $15 \mathrm{~min}$ by $1 \%$ formaldehyde, and the cross-linking was terminated with $125 \mathrm{mM}$ glycine. Alternatively DEC1 stably transfected cells were cultured in the presence of tetracycline 
$(0.1 \mu \mathrm{g} / \mathrm{ml})$ for $24 \mathrm{~h}$ and then processed as described for HepG2 cells. The soluble chromatins were prepared, pre-cleared with protein $\mathrm{G}$ beads and incubated with an Flag antibody (HepG2) or anti-DEC1 (stably transfected cells). As a control, the antibody was replaced with pre-immune IgG. The antibody-bound chromatins, DNA input (1/20 of the antibody-bound chromatins) as well as lgG-control were analyzed by PCR for the presence of the genomic fragment containing the E-box of interest, other E-boxes or no E-box. Both cells produced similar images and the image from HepG2 cells is shown).

Fig. 3. Inverse expression between DEC1 and SHP (A) Suppressed expression of SHP by DEC1 HepG2 cells were seeded in 8 -well chamber slides $\left(2 \times 10^{4}\right.$ cells/ well) and cultured for $24 \mathrm{~h}$. Cells were then transfected with Flag-DEC1 (500 ng) or the vector by TransfeX reagent. The transfected cells were cultured for another $24 \mathrm{~h}$ and subsequently fixed with $4 \%$ paraformaldehyde for $10 \mathrm{~min}$ at $\mathrm{pH}$ 7.4. Cells were permeabilized with $0.1 \%$ Triton $X-100$ for 10 min. Chamber slides were incubated with $1 \%$ BSA $(2 \mathrm{mg} / \mathrm{mL})$ for $1 \mathrm{~h}$ then incubated overnight with antiSHP antibody or anti-Flag antibody. The anti-SHP antibody was located by Alexa Fluor ${ }^{\circledR} 488$ conjugated goat anti-rabbit IgG (green), whereas the anti-Flag antibody with Alexa Fluor ${ }^{\circledR} 555$ conjugated goat anti-mouse IgG (red). The slides were then mounted with ProLong Gold Antifade Mountant with DAPI (Blue). Cells were then imaged with confocal microscope. To provide more quantitative information, same experiments were performed in 6-well plates and cell lysates were prepared. Lysates $(\underline{10} \mu \mathrm{g})$ were analyzed by Western blotting for the expression of SHP and DEC1 (Right panel). (B) Regulated expression of DEC1 and SHP by valproate in serum-shocked circadian induction HepG2 cells were seeded in 6- or 24-well plates at a density of $6 \times 10^{5}$ or $1.5 \times 10^{5}$ cells/well and 
cultured to reach $75 \%$ confluency. Cells were then shocked with media containing $50 \%$ horse serum for $2 \mathrm{~h}$. Thereafter, the shocked cells were cultured in normal media or the same media containing valproate at $2 \mathrm{mM}$. Cells were harvested at a $6 \mathrm{~h}$ interval. The expression of DEC1 and SHP was determined by RT-qPCR with Taqman probes. 
A

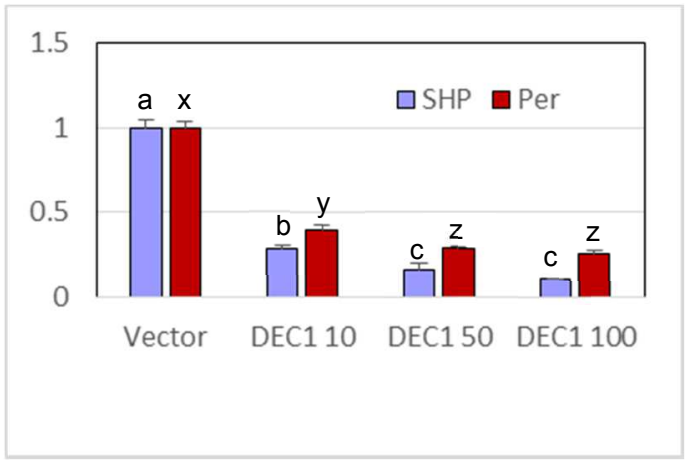

B

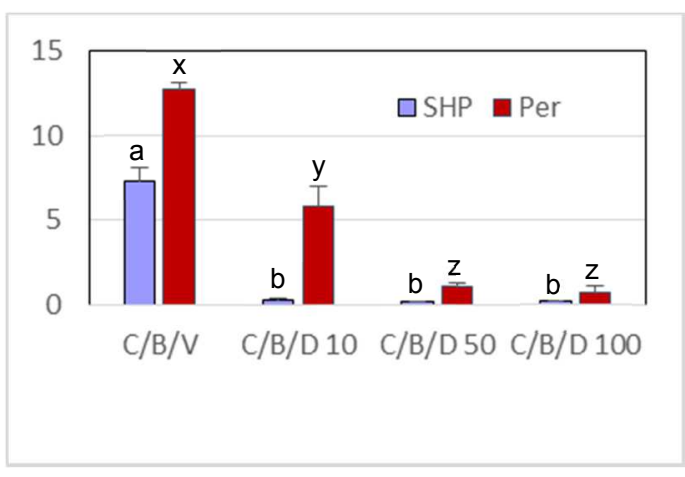

C

DEC1

B HLH
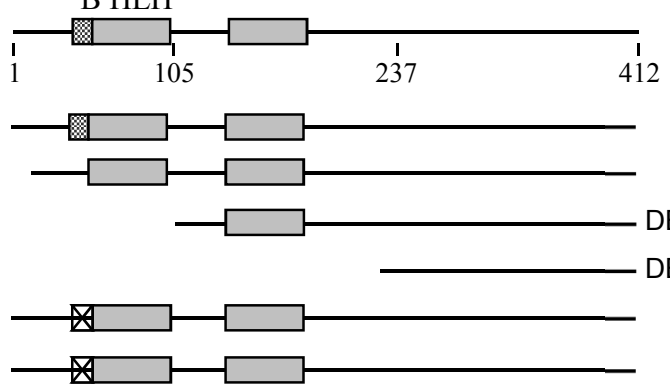

EC1(237-412

$\mathrm{DEC} 1_{\mathrm{P} 5 \mathrm{E}}$

DEC $1_{\text {R58 }}$

Relative luciferase activity

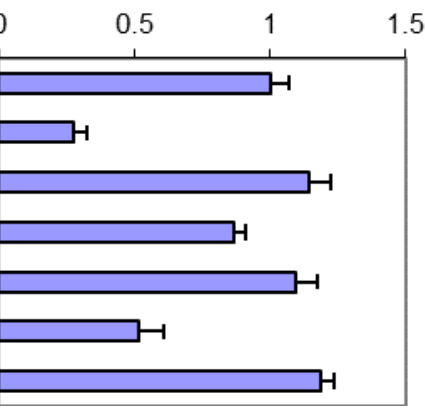

Fig. 1 
A

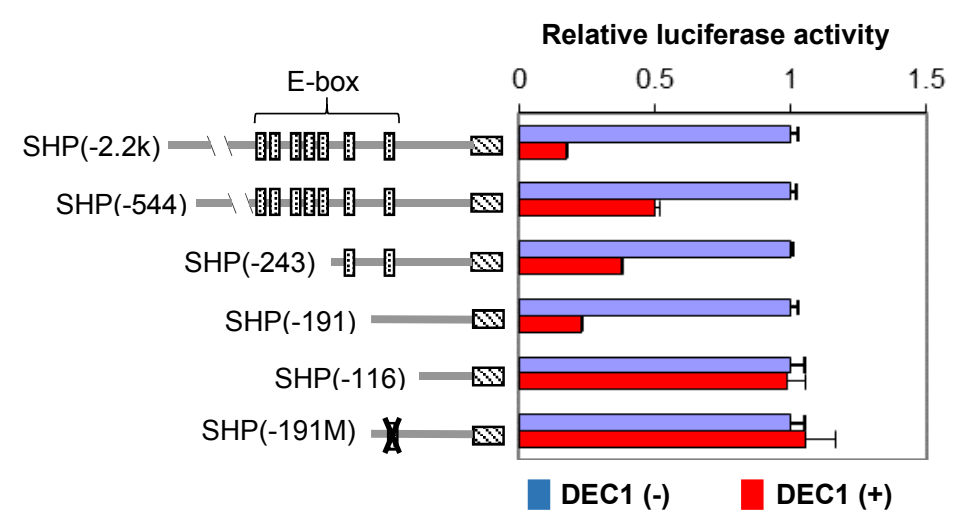

B

\begin{tabular}{|c|c|c|c|c|c|c|c|c|c|c|c|}
\hline DEC1 nuclear extract & $\sqrt{ }$ & $\sqrt{ }$ & $\sqrt{ }$ & $\sqrt{ }$ & $\sqrt{ }$ & $\sqrt{ }$ & $\sqrt{ }$ & $\sqrt{ }$ & $\sqrt{ }$ & $\sqrt{ }$ & $\mathrm{n}$ \\
\hline Biotin-probe (E1) & $\sqrt{ }$ & $\sqrt{ }$ & $\sqrt{ }$ & $\sqrt{ }$ & $\sqrt{ }$ & $\sqrt{ }$ & $\sqrt{v}$ & $\sqrt{ }$ & $\mathrm{v}$ & $\sqrt{ }$ & v \\
\hline DEC1 antibody & & $\sqrt{ }$ & $\sqrt{ }$ & & & & & & & & \\
\hline Competing E1 or E1m & & & & & $\sqrt{ }$ & $\sqrt{ }$ & & & & & QEEC1 antibody \\
\hline Other E-box competing & & & & & & & $\sqrt{ }$ & $\sqrt{ }$ & $\mathrm{v}$ & $\sqrt{ }$ & \\
\hline
\end{tabular}

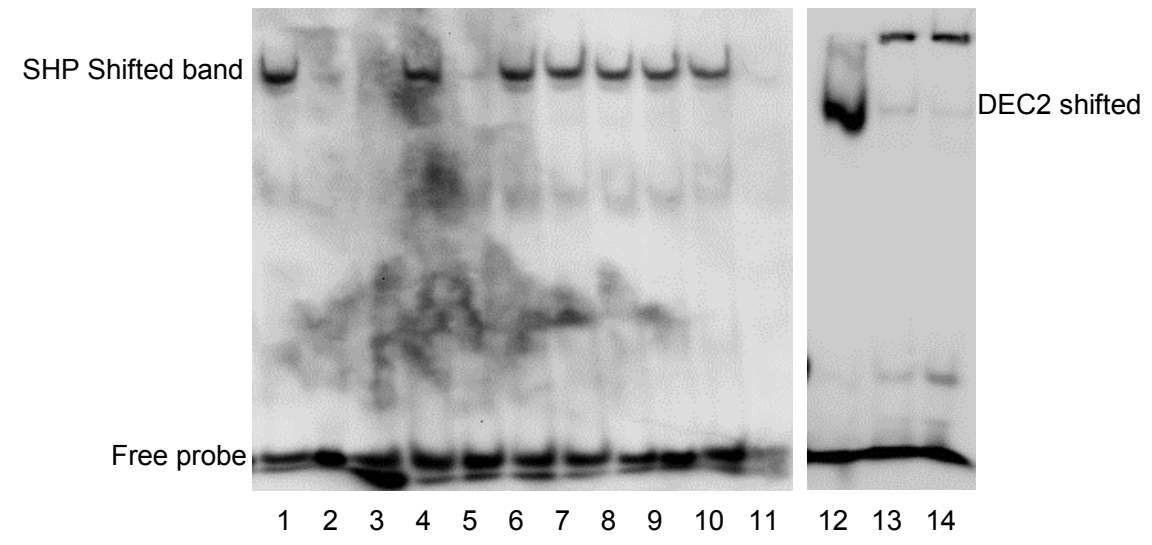

C
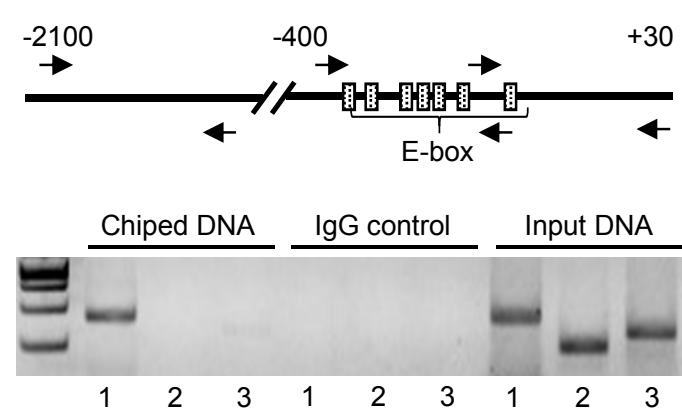

$1=$ BE-box $(-237 /+33$, binding E-box $)$ 2 = OE-box (-377/-186, other E-boxes) 3 = NE-box (-2061/-1820, no E-box)

Fig. 2 
A

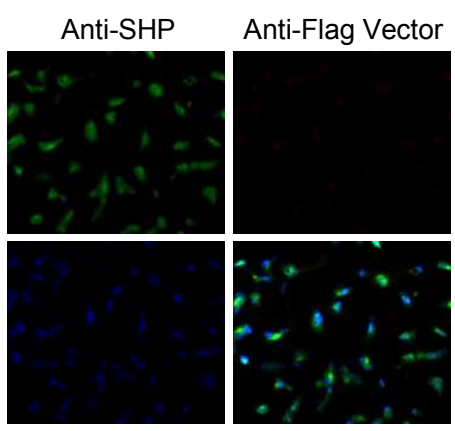

DAPI

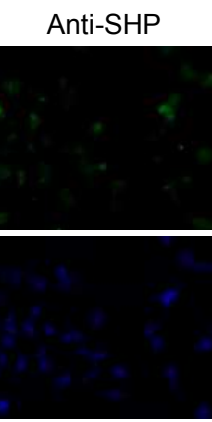

DAPI

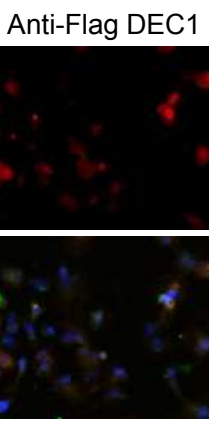

Merged
Transfection

Vector DEC1

Anti-Flag (DEC1)

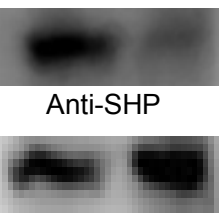

Anti-GAPDH

B

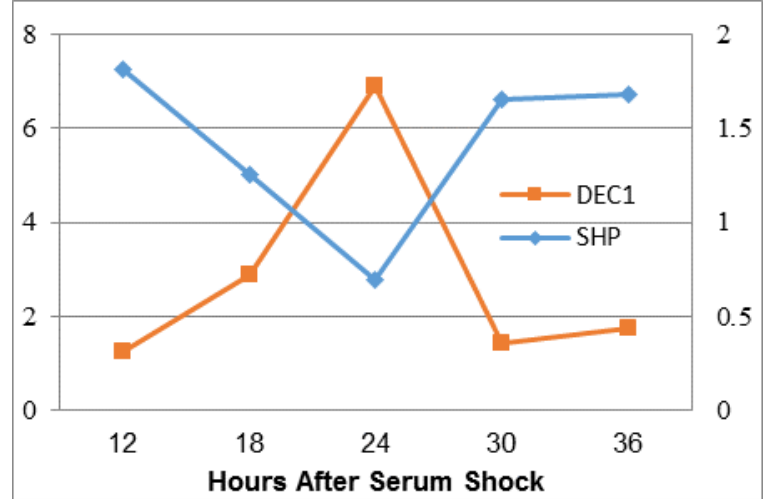

Fig. 3 